\title{
Posterior Fossa Ependymoma
}

\author{
M. Sean Grady, M.D. ${ }^{1}$ \\ Kevin D. Judy, M.D. ${ }^{2}$ \\ Douglas C. Bigelow, M.D. ${ }^{2}$ \\ Grant P. Sinson, M.D. ${ }^{2}$ \\ Robert Rostomily, M.D. ${ }^{3}$ \\ H. Richard Winn, M.D. ${ }^{3}$
}

\section{CASE HISTORY}

A 60-year-old female with chronic lymphocytic leukemia sought treatment after experiencing double vision for 3 weeks. She also noted difficulty with her balance and ambulation. She was evaluated by her oncologist who referred her for magnetic resonance (MR) imaging (Fig. 1). On physical examination, she was alert and oriented. Her speech was clear. Her extraocular muscles were intact, her tongue was midline, her hearing was grossly normal, and the remainder of her cranial nerves were intact. She had pronounced dysmetria on the right side. In addition, the patient had a positive Romberg's sign as well as an ataxic gait.

\section{RESPONSES}

This 60-year-old woman has a posterior fossa tumor, which presented primarily with symptoms referable to the right cerebellum. Her imaging studies show a large tumor extending through the foramen of Luschka, located laterally in the cerebellum. Undoubtedly, there is cranial nerve displacement by the tumor, most likely anterior and lateral.
Typically, ependymomas are somewhat vascular. The signal voids within this particular tumor (Fig. 1B) raise my concern about intraoperative bleeding obscuring the visual field under the microscope. I think that it is unlikely that an ependymoma could be embolized, but I think that I would recommend cerebral angiography before surgery to see if it would be possible.

\section{SURGICAL MANAGEMENT}

Several surgical approaches, including a retromastoid suboccipital craniotomy or a transmastoid presigmoid approach, could be contemplated. Computed tomography would demonstrate the degree of aeration in the mastoid region and help determine the ease of the latter approach. I would choose a retromastoid suboccipital craniotomy, placing the patient in a lateral position with the head turned downward and flexed to orient the petrous bone vertically. During bone removal, I would expose the entire sigmoid sinus so that the approach could be as lateral as possible. Intraoperatively, I would definitely monitor brain stem and auditory evoked potentials as well as the facial and hypoglossal nerves.

After the craniotomy, the cerebellum will need to be retracted. If relaxation is insufficient, re-

Skull Base, volume 11, number 3, 2001. 'Department of Neurosurgery and ${ }^{2}$ Cranial Base Surgery Center, University of Pennsylvania Medical Center, Philadelphia, Pennsylvania; ${ }^{3}$ Department of Neurological Surgery, Harborview Medical Center, University of Washington, Seattle, Washington. Copyright (c) 2001 by Thieme Medical Publishers, Inc., 333 Seventh Avenue, New York, NY 10001, USA. Tel: +1(212) 584-4662. 1531-5010,p;2001,11,03,219,224,ftx,en;sbs00225x. 
section of a lateral component of the cerebellum could be considered. Next, I would debulk the tumor using an ultrasonic aspirator with cautery incorporated into the tool to control bleeding. I would incorporate frameless stereotaxy to assist in removal of the tumor to gauge when I would be approaching the brain stem. Because I suspect that the tumor is displacing the cranial nerves anteriorly, I would expect to encounter these nerves after I had debulked the tumor somewhat and could begin sharp microdissection of the nerves away from the tumor maintaining a plane within the arachnoid if possible.

Typically, a margin can be identified with ependymomas although imaging studies in this case suggest that a well-defined plane may be difficult to achieve. If a gross total resection cannot be achieved, the patient will need postoperative radiation.

\section{Sean Grady, M.D. Department of Neurosurgery University of Pennsylvania Medical Center Fifth Floor Silverstein Pavilion 3400 Spruce Street Philadelphia, Pennsylvania 19104-4283 E-mail: gradys@uphs.upenn.edu}

The magnetic resonance (MR) images represent a $3-\mathrm{cm}$ ependymoma that has extended from the fourth ventricle through the left foramen of Luschka all the way to the cerebellopontine angle (CPA) and down to the foramen magnum. There is no known association between chronic lymphocytic leukemia and ependymomas. Unfortunately, this woman appears to have two primary neoplasms.

The first consideration should be a preoperative evaluation for the ependymoma. The MR images suggest evidence of hydrocephalus, which we would treat prophylactically at surgery with a ventriculostomy. The ventriculostomy would serve to (1) reduce the pressure on the posterior fossa and (2) permit collection of cerebrospinal fluid (CSF) for cytology before the tumor was touched. The latter is necessary to determine if the patient's ependymoma has spread. Before surgery, the patient should undergo a complete spinal MR imaging study to rule out drop metastases. Obtaining the spinal MR images and the CSF cytology before surgery would help optimize postoperative treatment decisions.

\section{SURGICAL MANAGEMENT}

The standard neurosurgical approach would be a midline suboccipital craniotomy, splitting the vermis, entering the fourth ventricle, and resecting the tumor. This approach, however, would damage a significant amount of tissue. A second standard neurosurgical approach would be to perform a lateral suboccipital craniotomy for tumor of the CPA. Unfortunately, the ependymoma is intrinsic and not extra-axial like an acoustic neuroma. The surgeon would have to resect at least part of the normal left cerebellar hemisphere to gain exposure to the tumor.

We would favor a more lateral approach combining a retrolabyrinthine exposure with a transcondylar approach to the foramen magnum. An anteriorly based C-shaped incision well posterior of the auricle would be made. The vertebral artery would be identified and dissected from $\mathrm{C} 1$ to its dural entry. Burr holes would be placed adjacent to the left transverse sinus, and a suboccipital flap would be turned. The exposure would be extended by removing the bone down to the foramen magnum, including the posterior half of the occipital condyle. The temporal bone would be drilled up to the semicircular canals, and the retrofacial air cells would be opened to the jugular bulb to optimize the exposure while preserving hearing. If more anterior exposure were needed, the posterior semicircular canal could be plugged and sacrificed; however, the risk to hearing would increase substantially.

The dura would be incised anterior to the sigmoid sinus from the petrosal sinus down to the 
A
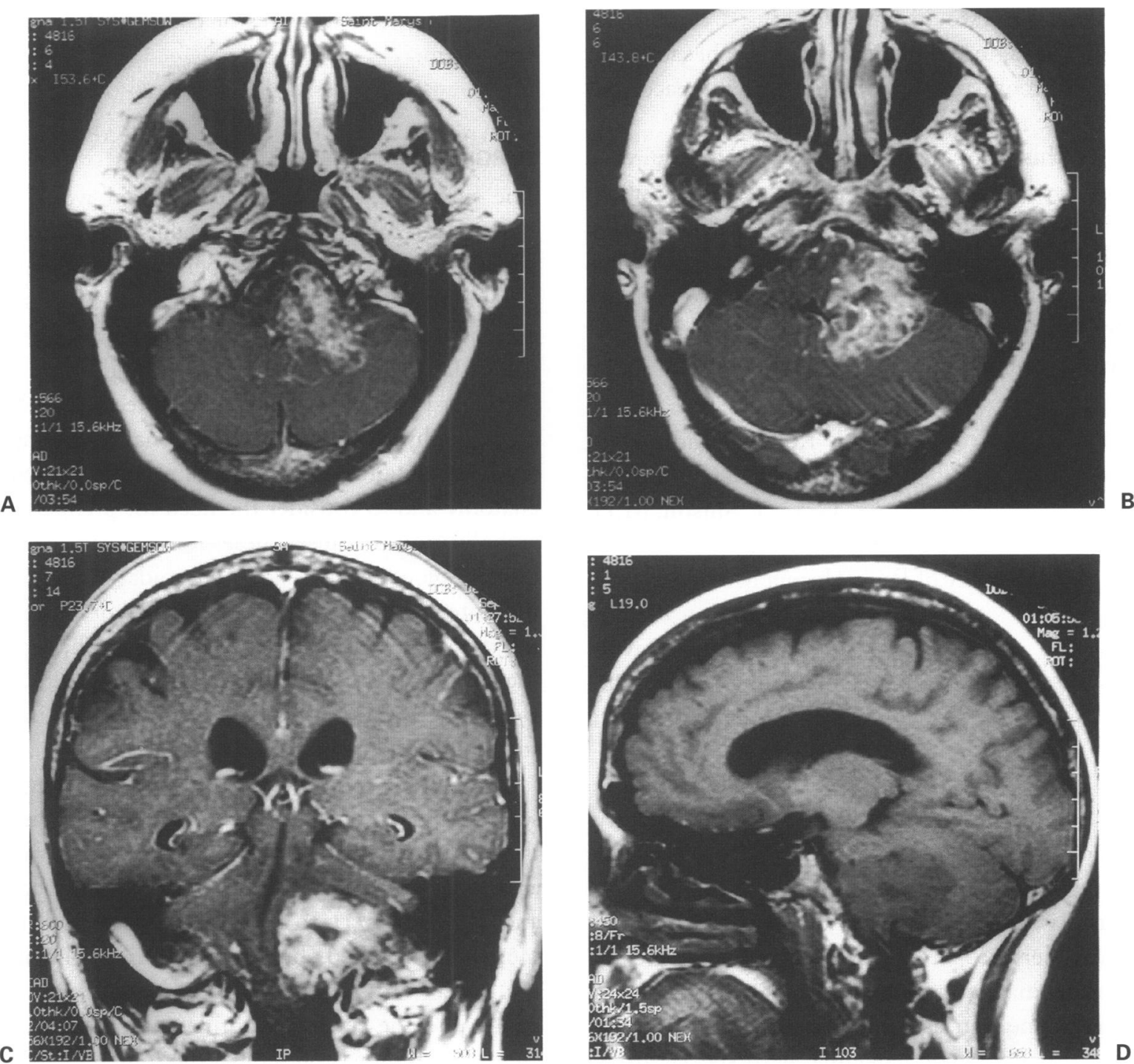

Figure 1 (A and B) Axial (at two different levels), (C) coronal, and (D) sagittal magnetic resonance images of a large ependymoma extending through and dilating the foramen of Luschka on the left.

jugular bulb. The superior petrosal sinus would be ligated and the tentorium incised so that the sigmoid sinus could be reflected posteriorly. This maneuver would provide excellent exposure of the lateral aspect of the tumor and allow visualization of the cranial nerves, which would be monitored intraoperatively. The dura would also be opened posterior to the sigmoid sinus down to the vertebral artery to gain access to the inferior aspect of the tumor at the foramen magnum.

If this lesion is indeed a low-grade ependymoma, its margins would be followed back up into the fourth ventricle. Approaching the tumor from a lateral exposure such as this would minimize risks to the surrounding normal cerebellum and brain stem. The Cavitron ultrasonic aspirator (Valleylab; 
Boulder, $\mathrm{CO}$ ) would have to be used carefully to avoid damaging the cranial nerves because the working room through the very small spaces between the nerves would be limited.

This extremely unusual tumor requires a more aggressive approach than a standard suboccipital craniotomy. In adults ependymomas tend to be low grade so that one could expect to achieve a gross total resection of this tumor. If the patient's CSF cytology and spinal MR images were negative, she would require no further therapy.

Kevin D. Judy, M.D. Douglas C. Bigelow, M.D. Grant P. Sinson, M.D.

Center for Cranial Base Surgery University of Pennsylvania Medical Center 3400 Spruce Street 5 Silverstein/Ravdin Philadelphia, Pennsylvania 19104-4283 E-mail: bigs@mail.med.upenn.edu

This case demonstrates a large eccentric enhancing ependymoma in the posterior fossa involving the foramen of Luschka in a 60-year-old woman with a history of chronic lymphocytic leukemia. Her preoperative evaluation should include a staging magnetic resonance (MR) imaging study of her spine and assessment of the status of her chronic lymphocytic leukemia, with special attention to her hematologic status. The following discussion assumes that her chronic lymphocytic leukemia is stabilized, that her long-term prognosis is good, and that her hematologic status is adequate. Thus, a radical resection should be the goal of surgery because most studies support the prognostic importance of as complete a resection as possible. Of course, in determining the value of aggressive resection, the surgeon must consider the negative impact of metastatic disease on survival on preoperative staging studies and the possible morbidity associated with resection when tumor is densely adherent to cranial nerves or coats or invades the brain stem.

\section{SURGICAL MANAGEMENT}

The surgical planning for this patient would include a careful preoperative evaluation of cranial nerve function and intraoperative electromyographic monitoring of the facial nerve, brain stem auditory evoked potentials, and lower cranial nerves (X, XI, XII). The patient would be treated with Decadron for several days before surgery. MR angiography or possibly conventional angiography would be obtained to review the pertinent vascular anatomy and to consider the need for preoperative embolization because these lesions can be extremely bloody. Most likely, choroidal branches of the anterior inferior cerebellar artery or posterior inferior cerebellar artery (PICA), or both, supply the tumor. Given the patient's hydrocephalus, we would plan a ventriculostomy as the first step in the surgical procedure.

The anatomic location of this lesion suggests several options for the surgical approach. However, the extensive nature of the tumor limits each single approach, and the need for multiple approaches and staged surgery to achieve complete resection must be considered. The tumor primarily involves the lateral recess and foramen of Luschka and expands into the cerebellopontine angle (CPA), but almost no tumor is present in the fourth ventricle. The tumor also involves the cerebellar hemisphere and cerebellar peduncle, and extends inferiorly near the level of the foramen magnum.

Thus, the surgical approach must address the lower pole of the tumor, providing access to the lateral recess and exposure for resection of the deeper medial portion. We would consider approaching this lesion by a transcerebellomedullary fissure, lateral retrosigmoid suboccipital, or presigmoid petrosal approach. The transcerebellomedullary fissure approach allows access to the inferior pole of the lesion, the lateral recess, and the blood 
supply from the PICA. Its major disadvantage is limited access to the medial and deep portion of the tumor. If complete resection cannot be obtained with this approach, a second-stage approach could be performed. Alternatively, by placing the patient in a three-quarter prone position, the transcerebellomedullary fissure approach could be combined with a suboccipital approach to gain better medial exposure. Although the suboccipital approach provides the best view of the CPA, access to the deeper and medial portions of the lesion might require extensive retraction and/or lateral cerebellar resection. The presigmoid petrosal approach would give the best view of the medial and deep portion of the lesion. However, to gain good exposure of the lower pole and inferior lateral recess, it might be necessary to combine the presigmoid petrosal approach with a suboccipital that extends inferiorly to the foramen magnum. Thus, the transcerebellomedullary fissure approach and suboccipital approach or presigmoid petrosal approach and suboccipital approach could be combined during one surgical event. We would favor the former with the expectation that the transcerebellomedullary fissure approach alone would probably permit most of the lesion to be resected.

Robert Rostomily, M.D.

H. Richard Winn, M.D.

Department of Neurological Surgery

University of Washington

Seattle, Washington

E-mail: mconnell@u.washington.edu 